\title{
ANALYSIS OF ELECTRIC GRID SECURITY UNDER TERRORIST THREAT
}

\author{
Javier Salmeron \\ Kevin Wood \\ Operations Research Department, \\ Naval Postgraduate School, Monterey, CA 93943-5001 \\ Ross Baldick \\ Department of Electrical Engineering \\ University of Texas at Austin, Austin, TX, 78712-1084
}

\begin{abstract}
We describe new analytical techniques to help mitigate the disruptions to electric power grids caused by terrorist attacks. New bilevel mathematical models and algorithms identify critical system components (e.g., transmission lines, generators, transformers) by creating maximally disruptive attack plans for terrorists assumed to have limited offensive resources. We report results for standard reliability test networks to show that the techniques identify critical components with modest computational effort. Keywords: Power flow; Interdiction; Homeland security
\end{abstract}

\section{INTRODUCTION}

Electric power systems are critical to any country's economy and security. In the United States, the system's vulnerability to physical disruptions from natural disasters and other causes has long been recognized [1]. This vulnerability has increased in recent years because infrastructure has not expanded as quickly as demand has, thereby reducing the system's "cushion" against failed, destroyed, or otherwise unavailable system components [2]. The threat of human attacks on the system has become more serious, too. The Committee on Science and Technology for Countering Terrorism [3] states the problem succinctly: "The nation's electric power systems must clearly be made more resilient to terrorist attack." To help reach this goal, this paper describes new bilevel optimization models and solution techniques for analyzing the security and resilience of electrical power grids against disruptions caused by terrorist attacks. (These techniques could also help analyze a system's susceptibility to natural disasters, but we do not study this issue explicitly.)

We propose techniques to identify critical sets of a power grid's components, e.g., generators, transmission lines, and transformers, by identifying maximally disruptive, coordinated (nearly simultaneous) attacks on a grid, which a terrorist group might undertake. By studying how to attack power grids, we will ultimately understand how to make them less vulnerable. We report results for our techniques applied to reliability-benchmark networks.

We search for optimal attacks, i.e., a set of attacks that causes the largest possible disruption given posited offensive resources. By considering the largest possible disruptions, our proposed protection plans will be appropriately conservative. Actual terrorist "resources" will always be uncertain, so our techniques must be fast enough to quickly analyze a wide range of probable scenarios. We concern ourselves only with physical attacks on the power grid and neglect the issue of "cyber-attacks" on the controlling Supervisory Control and Data Acquisition (SCADA) infrastructure. The implicit assumption is that the SCADA infrastructure has been hardened. A discussion of the issues for reliable communication in this context is presented in [4].

The rest of this paper is organized as follows: Section II describes the mathematical formulation of our models and describes procedures to solve them. Section III reports results of those models and algorithms applied to two IEEE reliability test systems. Section IV provides conclusions and points out directions for future research.

\section{APPROACH}

\section{A. Overview}

Our approach to identifying critical system components first develops a network-interdiction model [5] to represent the optimal-attack problem that a terrorist group might face. This model is a max-min $(\mathrm{Mm})$ problem:

$$
\begin{aligned}
(\mathrm{Mm}) \quad \max _{\boldsymbol{\delta} \in \Delta} \min _{\mathbf{p}} \mathbf{c}^{T} \mathbf{p} & \\
\text { s.t. } \mathbf{g}(\mathbf{p}, \boldsymbol{\delta}) & \leq \mathbf{b} \\
\mathbf{p} & \geq \mathbf{0}
\end{aligned}
$$

An interdiction plan is represented by the binary vector $\boldsymbol{\delta}$, whose $k$-th entry $\delta_{k}$ is 1 if component $k$ of the system is attacked and is 0 otherwise. For a given plan, the inner problem is an optimal power-flow model [6, p. 514] that minimizes generation costs plus the penalty associated with unmet demand, together denoted by $\mathbf{c}^{T} \mathbf{p}$. Here, $\mathbf{p}$ represents power flows, generation outputs, phase angles and "unmet demand," i.e., the amount of load shed; c represents linearized generation costs, and the costs of unmet demand. The outer maximization chooses the most disruptive, resource-constrained interdiction plan $\delta \in \Delta$, where $\Delta$ is a discrete set representing attacks that a terrorist group might be able to carry out. In this model, $\mathbf{g}$ corresponds to a set of functions that are nonlinear in 
$(\mathbf{p}, \boldsymbol{\delta})$. The inner problem involves a simplified optimal power-flow model, with constraint functions $\mathbf{g}(\mathbf{p}, \boldsymbol{\delta})$ that are, however, linear in $\mathbf{p}$ for a fixed $\boldsymbol{\delta}=\hat{\boldsymbol{\delta}}$. The model extends easily to handle the cost of repairs.

For simplicity, we first describe a power-flow model that minimizes the instantaneous cost of system operation, including unmet demand, measured in $\$ / \mathrm{h}$. Power disruptions resulting from cascading outages immediately after attack are ignored, so we are actually measuring cost after initial restoration of outaged but undamaged equipment. Later in the paper we consider the cost of unmet demand after the initial restoration, recognizing that this cost will be changing over time as damaged equipment is repaired following an attack.

The remainder of this section explains the basic mathematical models and algorithms our research has developed. We first summarize our DC approximation of the AC power-flow model, and then show how to incorporate that approximation as part of an interdiction model. Finally, we introduce a heuristic algorithm for solving the combined power-flow and interdiction model.

\section{B. Power-flow model}

We approximate active power flows with a DC model, denoted DC-OPF (DC-Optimal Power Flow), which neglects reactive power effects and nonlinear losses. This approximation is normally acceptable in the context of long-term, "coarse-grained" security analysis [6, p. 419]. In the eventual min-max interdiction model, DC-OPF will be modified through a set of interdiction variables.

\section{Indices and index sets: \\ $i \in \mathbf{I}$ buses \\ $g \in \mathbf{G}$ generating units \\ $l \in \mathbf{L} \quad$ transmission lines \\ $c \in \mathbf{C}$ consumer sectors \\ $s \in \mathbf{S} \quad$ substations \\ $i \in \mathbf{I}_{s} \quad$ buses at substation $s$ \\ $g \in \mathbf{G}_{i} \quad$ generating units connected to bus $i$ \\ $l \in \mathbf{L}_{i}^{\text {Bus }}$ lines connected to bus $i$ \\ $l \in \mathbf{L}_{s}^{\text {Sub }}$ lines connected to substation $s$ (including transformers, which are represented by lines) \\ $l^{\prime} \in \mathbf{L}_{l}^{\text {Par }}$ lines $l^{\prime} \neq l$ running in parallel to line $l$}

\section{Parameters (units):}

$o(l), d(l)$ : origin and destination buses of line $l$; more than one line with the same $o(l), d(l)$ may exist

$i(g) \quad$ bus for generator $g$, i.e., $g \in \mathbf{G}_{i(g)}$

$d_{i c} \quad$ load of consumer sector $c$ at bus $i$ (MW)

$\bar{P}_{l}^{\text {Line }} \quad$ transmission capacity for line $l(\mathrm{MW})$

$\bar{P}_{g}^{G e n} \quad$ maximum output from generator $g(\mathrm{MW})$ $r_{l}, x_{l} \quad$ resistance, reactance of line $l(\Omega)$. (We assume $\left.x_{l} \gg>r_{l}\right)$; series susceptance is $B_{l}=x_{l} /\left(r_{l}^{2}+x_{l}^{2}\right)$

$h_{g} \quad$ generation cost for unit $g(\$ / \mathrm{MWh})$

$f_{i c} \quad$ load-shedding cost for customer sector $c$ at bus $i$ (\$/MWh)

\section{Decision variables (units):}

$P_{g}^{G e n} \quad$ generation from unit $g(\mathrm{MW})$

$P_{l}^{\text {Line }} \quad$ power flow on line $l$ (MW)

$S_{i c} \quad$ load shed by customer sector $c$ at bus $i$ (MW)

$\theta_{i} \quad$ phase angle at bus $i$ (radians)

\section{Formulation of DC-OPF:}

(Remark: All units above are converted into per-unit values for a base load of 100MW.)

$\min _{\mathbf{P}^{G e n}, \mathbf{P}^{i n e}, \mathbf{S}, \mathbf{\theta}} \sum_{g} h_{g} P_{g}^{G e n}+\sum_{i} \sum_{c} f_{i c} S_{i c}$

subject to:

$P_{l}^{\text {Line }}=B_{l}\left(\theta_{o(l)}-\theta_{d(l)}\right) \quad \forall l$

$\sum_{g} P_{g}^{G e n}-\sum_{l \mid o(l)=i} P_{l}^{\text {Line }}+$

$\sum_{l \mid d(l)=i} P_{l}^{\text {Line }}=\sum_{c}\left(d_{i c}-S_{i c}\right) \quad \forall i$

$-\bar{P}_{l}^{\text {Line }} \leq P_{l}^{\text {Line }} \leq \bar{P}_{l}^{\text {Line }} \quad \forall l$

$0 \leq P_{g}^{G e n} \leq \bar{P}_{g}^{G e n} \quad \forall g$

$0 \leq S_{i c} \leq d_{i c} \quad \forall i, c$.

The objective of DC-OPF (DC.0) is to minimize generating plus shedding costs measured in $\$ / \mathrm{h}$. Constraints (DC.1) approximate active power flows on the lines. Constraints (DC.2) maintain power balance at the buses. Constraints (DC.3) and (DC.4) set maximum line power flows and generating-unit outputs. Minimum power outputs are set to zero for all generating units for simplicity here, but extensions to non-zero minima are straightforward. Constraints (DC.5) state that load shedding cannot exceed demand. A version of DC-OPF will be a subproblem of the interdiction model described next.

\section{Interdiction model}

"The interdictor" in our model, a group of terrorists, will make a coordinated set of resource-constrained interdictions (attacks) on the power grid. We make the following assumptions on the effect of each interdiction:

- Line interdiction: All lines running physically in parallel at the point of an attack are opened. (Typically, these lines are mounted on the same towers, and an attack on one is an attack on all.)

- Transformer interdiction: The line representing the transformer is opened.

- Generator interdiction: The generator is disconnected from the grid. 
- Bus interdiction: All lines, generation, and load connected to the bus are disconnected.

- Substation interdiction: All buses at the substation are disconnected; this triggers the corresponding bus-interdiction effects just described.

Terrorist resource constraints can accommodate information from intelligence sources, whether it is specific as we shall assume, or generic, such as "any three attacks might happen." For demonstration purposes, we model this feature through a simple knapsack constraint.

\section{Additional sets and parameters required:}

$\mathbf{G}^{*} \subseteq \mathbf{G}, \mathbf{L}^{*} \subseteq \mathbf{L}, \mathbf{I}^{*} \subseteq \mathbf{I}, \mathbf{S}^{*} \subseteq \mathbf{S}$ : interdictable generators, lines, buses, and substations, respectively. These are "interdictable components."

$M_{g}^{\text {Gen }}, M_{l}^{\text {Line }}, M_{i}^{\text {Bus }}, M_{s}^{\text {Sub }}$ : resource required to interdict generator $g$, line $l$, bus $i$, and substation $s$, respectively.

$M \quad$ total interdiction resource available to terrorists.

\section{Interdiction variables:}

$\delta_{g}^{\text {Gen }}, \delta_{l}^{\text {Line }}, \delta_{i}^{\text {Bus }}, \delta_{s}^{\text {Sub }}$ : binary variables that take the value 1

if generator $\mathrm{g}$, line $l$, bus $i$ or substation $s$, respectively, are interdicted, and are 0 otherwise.

\section{Formulation of I-DC-OPF:}

$\max _{\boldsymbol{\delta}^{\text {Gen }}, \boldsymbol{\delta}^{\text {Line }}, \boldsymbol{\delta}^{\text {bus }}, \mathbf{\delta}^{\text {sub }}} \gamma\left(\boldsymbol{\delta}^{\text {Gen }}, \boldsymbol{\delta}^{\text {Line }}, \boldsymbol{\delta}^{\text {Bus }}, \boldsymbol{\delta}^{\text {Sub }}\right)$

subject to:

$$
\begin{aligned}
\sum_{g \in \mathrm{G}^{*}} M_{g}^{\text {Gen }} & \delta_{g}^{\text {Gen }}+\sum_{l \in \mathrm{L}^{*}} M_{l}^{\text {Line }} \delta_{l}^{\text {Line }}+ \\
& \sum_{i \in \mathrm{I}^{*}} M_{i}^{\text {Bus }} \delta_{i}^{\text {Bus }}+\sum_{s \in S^{*}} M_{s}^{\text {Sub }} \delta_{s}^{\text {Sub }} \leq M
\end{aligned}
$$

All variables $\boldsymbol{\delta}$ are binary, but are fixed to 0 if not associated with $\mathbf{G}^{*}, \mathbf{L}^{*}, \mathbf{I}^{*}$, or $\mathbf{S}^{*}$

And where:

$$
\begin{aligned}
& \gamma\left(\boldsymbol{\delta}^{G e n}, \boldsymbol{\delta}^{L i n e}, \boldsymbol{\delta}^{B u s}, \boldsymbol{\delta}^{S u b}\right)= \\
& \min _{\mathbf{p}^{G e n,}, \mathbf{p}^{\text {Line }, \mathbf{S}, \boldsymbol{\theta}}} \sum_{g} h_{g} P_{g}^{G e n}+\sum_{i} \sum_{c} f_{i c} S_{i c}
\end{aligned}
$$

subject to:

(Note: Nonlinear constraints in $\boldsymbol{\delta}$ are used for conciseness below; linear replacements are straightforward.)

$$
\begin{aligned}
& P_{l}^{\text {Line }}=B_{l}\left(\theta_{o(l)}-\theta_{d(l)}\right)\left(1-\delta_{l}^{\text {Line }}\right)\left(1-\delta_{o(l)}^{\text {Bus }}\right)\left(1-\delta_{d(l)}^{\text {Bus }}\right) \\
& \prod_{s \mid l \in \mathrm{L}_{s}^{\text {sub }}}\left(1-\delta_{s}^{\text {Sub }}\right) \prod_{l^{\prime} l^{\prime} \in \mathrm{L}_{l}^{P_{a r}}}\left(1-\delta_{l^{\prime}}^{\text {Line }}\right) \quad \forall l \\
& \sum_{g} P_{g}^{\text {Gen }}-\sum_{l \mid o(l)=i} P_{l}^{\text {Line }}+\sum_{l \mid d(l)=i} P_{l}^{\text {Line }}= \\
& \sum_{c}\left(d_{i c}-S_{i c}\right) \\
& \forall i
\end{aligned}
$$

$$
\begin{aligned}
& -\bar{P}_{l}^{\text {Line }}\left(1-\delta_{l}^{\text {Line }}\right)\left(1-\delta_{o(l)}^{\text {Bus }}\right)\left(1-\delta_{d(l)}^{\text {Bus }}\right) \\
& \prod_{s \mid l \in \mathrm{L}_{\mathrm{s}}^{\text {sub }}}\left(1-\delta_{s}^{\text {Sub }}\right) \prod_{l \mid l l^{\prime} \in \mathbf{L}_{l}^{a r}}\left(1-\delta_{l^{\prime}}^{\text {Line }}\right) \leq P_{l}^{\text {Line }} \leq \\
& \bar{P}_{l}^{\text {Line }}\left(1-\delta_{l}^{\text {Line }}\right)\left(1-\delta_{o(l)}^{\text {Bus }}\right)\left(1-\delta_{d(l)}^{\text {Bus }}\right) \\
& \prod_{s l l \mid \mathbf{L}_{s}^{s u b}}\left(1-\delta_{s}^{\text {Sub }}\right) \prod_{l^{\prime} l^{\prime} \in \mathbf{L}_{l}^{\mathbf{L}_{l}^{a r}}}\left(1-\delta_{l^{L}}^{\text {Line }}\right) \quad \forall l \\
& 0 \leq P_{g}^{G e n} \leq\left(1-\delta_{i(g)}^{B u s}\right)\left(1-\delta_{g}^{G e n}\right) \bar{P}_{g}^{G e n} \quad \forall g \\
& 0 \leq S_{i c} \leq d_{i c} \quad \forall i, c \text {. }
\end{aligned}
$$

I-DC-OPF maximizes generation costs plus load-shedding costs, which we refer to as "disruption." (A more intuitive definition would also subtract nominal generation cost, but this is a constant factor we shall ignore.) Disruption is evaluated through the inner minimization problem that consists of the power-flow model DC-OPF with interdicted components removed. At the outer level, equation (I.1) reflects the terrorists' options to interdict different combinations of components in the grid without exceeding their resources. Restrictions (I.2) define terrorist actions as binary variables and ensure non-interdiction of certain grid components (e.g., hardened components).

Equations (IDC.1)-(IDC.5) are analogs of (DC.1)(DC.5). Here, however, the components that have been interdicted, directly or indirectly, are removed from the equations through the binary interdiction variables. For example, if some line $l$ is connected to an interdicted substation $s$, i.e., $\delta_{s}^{S u b}=1$, then constraints (IDC.1) and (IDC.3) for line $l$ yield $P_{l}^{\text {Line }}=0$.

The computational challenge of I-DC-OPF stems from the max-min structure of the problem. The optimal objective value of the linearized version of the inner minimization, as a function of continuous $\boldsymbol{\delta}$, is convex. Hence, I-DC-OPF involves the maximization of a convex function, which is usually a difficult task.

\section{Interdiction algorithm}

Future research will investigate the conversion of I-DC-OPF to a linear mixed-integer program which could be solved directly or through decomposition [7]. At this juncture, we have devised a decomposition-based heuristic to obtain acceptable interdiction plans (for the terrorists), although not necessarily optimal ones.

The algorithm begins by solving DC-OPF, "the subproblem," assuming no attacks. The result is an optimal power flow for normal operations, a flow that should minimize generation costs without shedding any load. The power-flow pattern is used to assign relative values (see below) to all the components of the power grid. Next, the algorithm solves a "master problem" to identify an interdiction plan that maximizes the estimated value of interdicted assets while not exceeding available interdiction resources. With this plan, the constraints of DC-OPF are modified and the new subproblem solved. The result is a 
power flow that minimizes generation costs plus the penalty associated with load shedding, given the new interdictions. Typically, some load will be shed in the new solution since valuable assets have been removed from the grid.

The process continues by finding alternative interdiction plans and by evaluating load shedding for each of them. This algorithm may be viewed as a heuristic version of Benders decomposition [7] to solve the bilevel program, I-DC-OPF. The master problem incorporates super-valid inequalities (constraints that eliminate some solutions, but not all optimal ones, unless an optimal solution has already been identified [5]) to avoid repeating solutions. We next provide details of the two models required in the decomposition algorithm.

Subproblem: DC-OPF for a specific interdiction plan Assume that at iteration $t$ of our algorithm, a specific interdiction plan $\hat{\boldsymbol{\delta}}^{t}=\left(\hat{\boldsymbol{\delta}}^{\text {Gen,t }}, \hat{\boldsymbol{\delta}}^{\text {Line,t }}, \hat{\boldsymbol{\delta}}^{\text {Bus,t }}, \hat{\boldsymbol{\delta}}^{\text {Sub,t }}\right)$ is given. The power-flow model DC-OPF $\left(\hat{\boldsymbol{\delta}}^{t}\right)$, equations (IDC.0)(IDC.5), forms the subproblem and its solution yields objective value $\gamma\left(\hat{\boldsymbol{\delta}}^{t}\right)=\gamma\left(\hat{\boldsymbol{\delta}}^{\text {Gen, },}, \hat{\boldsymbol{\delta}}^{\text {Line,t }}, \hat{\boldsymbol{\delta}}^{\text {Bus }, t}, \hat{\boldsymbol{\delta}}^{\text {Sub }, t}\right)$ along with power flows, generation and unmet demand, which are represented by $\hat{\mathbf{P}}^{t}=\left(\hat{\mathbf{P}}^{\text {Line,t }}, \hat{\mathbf{P}}^{\text {Gen, } t}, \hat{\mathbf{S}}^{t}, \hat{\boldsymbol{\theta}}^{t}\right)$.

\section{Value estimates}

The solution $\hat{\mathbf{P}}^{t}=\left(\hat{\mathbf{P}}^{\text {Line }, t}, \hat{\mathbf{P}}^{\text {Gen }, t}, \hat{\mathbf{S}}^{t}, \hat{\boldsymbol{\theta}}^{t}\right)$, provided by $\operatorname{DC}-\mathrm{OPF}\left(\hat{\boldsymbol{\delta}}^{t}\right)$, serves to construct estimates of the attractiveness or "value" of components for further interdiction. To determine these estimates, we define a set of parameters which represent, essentially, estimated coefficients for a "Benders cut" that will be added to the master problem. (In this heuristic, a cut, or a set of aggregated cuts, is added to the master-problem objective function rather than being added as a constraint; the super-valid inequalities take the place of cuts that build up from iteration to iteration.) We first compute:

$$
\begin{aligned}
& F_{i}^{\text {Out }, t}=\sum_{\substack{l \mid o(l)=i \\
\wedge \hat{P}_{l}^{\text {Line }}>0}} \hat{P}_{l}^{\text {Line }, t}+\sum_{\substack{l \mid d(l)=i \\
\wedge \hat{P}_{l}^{\text {ine }}<0}}\left|\hat{P}_{l}^{\text {Line }, t}\right|, \text { flow out of bus } i \\
& F_{i}^{\text {Met }, t}=\sum_{c}\left(d_{i c}-\hat{S}_{i c}^{t}\right), \text { load supplied to bus } i .
\end{aligned}
$$

These totals are then used to compute:

$$
\begin{array}{rlrl}
V_{g}^{\text {Gen }, t} & =w^{\text {Gen }} \hat{P}_{g}^{\text {Gen }, t} & & \forall g \\
V_{l}^{\text {Line }, t} & =w^{\text {Line }}\left(\left|\hat{P}_{l}^{\text {Line }, t}\right|+\sum_{l^{\prime} \in \mathrm{L}_{l}^{\text {par }}}\left|\hat{P}_{l^{\prime}}^{\text {Line }, t}\right|\right) & \\
V_{i}^{\text {Bus }, t}=w^{\text {Bus }}\left(F_{i}^{\text {Met }, t}+F_{i}^{\text {Out }, t}\right) & & \forall i \\
V_{s}^{\text {Sub }, t}=w^{\text {Sub }} \sum_{l l l \in \mathrm{L}_{s}}\left|\hat{P}_{l}^{\text {Line }, t}\right| & \forall s
\end{array}
$$

The weights $w^{\text {Gen }}, w^{\text {Bus }}, w^{\text {Line }}$ and $w^{\text {Sub }}$ are specified by the user to reflect the relative importance of each type of component. Experience indicates that the algorithm is more efficient when using weights that provide higher incentives for attacks on buses and substations than on individual lines and generators, e.g., $w^{G e n}=2, \quad w^{B u s}=5$, $w^{\text {Line }}=1, w^{\text {Sub }}=5$.

We have found that the following modifications to the definition of value are useful, too: (a) The value for a component defined above is divided by the amount of resource required to interdict that component, to reflect value per unit of effort, (b) a minimum value $\varepsilon>0$ is always enforced, for reasons explained below, and (c) a running average of component values is used rather just the current iteration's values. Without the running average, if the power flow through a currently interdicted component is null, the asset will be unattractive in the immediately following iteration. This is counter-intuitive. (The running average could be computed in a variety of ways, but we use a simple arithmetic average over all iterations.)

\section{Master Problem: Finding a valuable interdiction plan}

Assume that a set of estimated values for each component of the grid, $\quad \mathbf{V}^{t}=\left(\mathbf{V}^{\text {Gen }, t}, \mathbf{V}^{\text {Line }, t}, \mathbf{V}^{\text {Bus }, t}, \mathbf{V}^{\text {Sub }, t}\right)$, has been calculated at iteration $t$; and, define the vector of previously generated interdiction plans $\hat{\Delta}^{t}=\left(\hat{\boldsymbol{\delta}}^{1}, \ldots, \hat{\boldsymbol{\delta}}^{t}\right)$. The interdiction master problem is then:

$$
\begin{aligned}
& \operatorname{MP}\left(\mathbf{V}^{t}, \hat{\Delta}^{t}\right): \max _{\substack{\delta^{G e n}, \delta^{\text {Line }} \\
\boldsymbol{\delta}^{\text {Bus }}, \mathbf{\delta}^{\text {sub }}}} \sum_{g \in \Gamma^{*}} V_{g}^{G e n, t} \delta_{g}^{G e n}+\sum_{l \in L^{*}} V_{l}^{\text {Line,t }} \delta_{l}^{\text {Line }}+ \\
& \sum_{i \in \mathrm{I}^{*}} V_{i}^{G e n, t} \delta_{i}^{G e n}+\sum_{s \in \mathrm{S}^{*}} V_{s}^{S u b, t} \delta_{s}^{S u b}
\end{aligned}
$$

subject to:

Eqs. (I.1) - (I.2) replacing $\boldsymbol{\delta}$ with $\boldsymbol{\delta}^{t}$

(MP.1) - (MP.2)

$\delta_{g}^{G e n}+\delta_{i}^{B u s} \leq 1 \quad \forall g \in \mathbf{G}_{i}^{*}, i \in \mathbf{I}^{*}$

$\delta_{l}^{\text {Line }}+\delta_{i}^{\text {Bus }} \leq 1 \quad \forall l \in \mathbf{L}_{i} \cap \mathbf{L}^{*}, i \in \mathbf{I}^{*}$

$\delta_{l}^{\text {Line }}+\delta_{l^{\prime}}^{\text {Line }} \leq 1 \quad \forall l^{\prime} \in \mathbf{L}_{l}^{\text {Par }} \cap \mathbf{L}^{*}, l \in \mathbf{L}^{*}$

$\delta_{i}^{\text {Bus }}+\delta_{s}^{\text {Sub }} \leq 1 \quad \forall i \in \mathbf{I}_{s} \cap \mathbf{I}^{*}, s \in \mathbf{S}^{*}$

$\delta_{l}^{\text {Line }}+\delta_{s}^{\text {Sub }} \leq 1 \quad \forall l \in \mathbf{L}_{s} \cap \mathbf{L}^{*}, s \in \mathbf{S}^{*}$

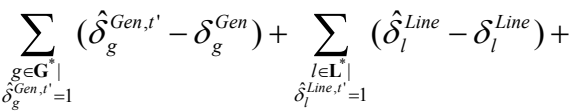

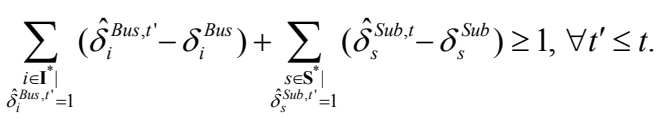

The solution to $\operatorname{MP}\left(\mathbf{V}^{t}, \hat{\Delta}^{t}\right)$ maximizes the estimated value of interdicted grid components. Constraints (MP.3) through (MP.7) serve the following purposes, respectively: Interdict a generator or the bus it is connected to, but not both; interdict a line or the bus it is connected to, but not both; if two lines run in parallel, interdict one line or the other, but not both (in the latter case, the interdiction of both lines simultaneously is actually represented by a single variable); interdict a bus or the substation it belongs to, but not both; and, interdict a line or the substation it is 
connected to, but not both. These constraints incorporate structural information about the system that the linear objective function cannot. Specifically, the constraints ensure that no system components are interdicted that are indirectly interdicted by attacks on other components. Finally, constraints (MP.8) ensure that the interdiction plan chosen at the incumbent iteration is different from all plans from previous iterations. These constraints assume that the optimal objective of the master problem always consumes a maximal amount of resource (i.e., insufficient resource remains to interdict any additional components). This is ensured by requiring every component maintain a minimum allowable value, $\varepsilon>0$.

$$
\text { Let } \hat{\boldsymbol{\delta}}^{t+1}=\left(\hat{\boldsymbol{\delta}}^{\text {Gen }, t+1}, \hat{\boldsymbol{\delta}}^{\text {Line }, t+1}, \hat{\boldsymbol{\delta}}^{\text {Bus }, t+1}, \hat{\boldsymbol{\delta}}^{\text {Sub }, t+1}\right) \text { denote the }
$$
solution to $\operatorname{MP}\left(\mathbf{V}^{t}, \hat{\Delta}^{t}\right)$. The vector $\hat{\boldsymbol{\delta}}^{t+1}$ is used in the subproblem to start a new iteration of the algorithm described next.

\section{I-ALG: Interdiction Algorithm}

Input: Grid data; Interdiction data; $T$ (iteration limit).

Output: $\hat{\boldsymbol{\delta}}^{*}$ is a feasible interdiction plan causing a disruption with cost $\gamma^{*}$. If the algorithm exits because $\operatorname{MP}\left(\mathbf{V}^{t}, \hat{\Delta}^{t}\right)$ is infeasible, then all feasible solutions have been enumerated, and $\hat{\boldsymbol{\delta}}^{*}$ is therefore optimal.

\section{Initialization:}

- $\quad$ Set $\hat{\boldsymbol{\delta}}^{1}=\left(\hat{\boldsymbol{\delta}}^{\text {Gen }, 1}, \hat{\boldsymbol{\delta}}^{\text {Line, } 1}, \hat{\boldsymbol{\delta}}^{\text {Bus }, 1}, \hat{\boldsymbol{\delta}}^{\text {Sub }, 1}\right) \leftarrow(\mathbf{0}, \mathbf{0}, \mathbf{0}, \mathbf{0})$ (initial attack plan).

- $\quad$ Set $\hat{\boldsymbol{\delta}}^{*} \leftarrow \hat{\boldsymbol{\delta}}^{1}$ (best plan so far) and $\hat{\Delta}^{1} \leftarrow\left\{\hat{\boldsymbol{\delta}}^{1}\right\}$.

- Set $\gamma^{*} \leftarrow 0$ (cost of the best plan so far).

- Set $t \leftarrow 1$.

\section{Subproblem:}

- Solve DC-OPF $\left(\hat{\boldsymbol{\delta}}^{t}\right)$ for objective value $\gamma\left(\hat{\boldsymbol{\delta}}^{t}\right)$ and solution $\hat{\mathbf{P}}^{t}=\left(\hat{\mathbf{P}}^{\text {Line, } t}, \hat{\mathbf{P}}^{\text {Gen }, t}, \hat{\mathbf{S}}^{t}, \hat{\boldsymbol{\theta}}^{t}\right)$.

- If $\gamma\left(\hat{\boldsymbol{\delta}}^{t}\right)>\gamma^{*}$ then $\gamma^{*} \leftarrow \gamma\left(\hat{\boldsymbol{\delta}}^{t}\right)$, and $\hat{\boldsymbol{\delta}}^{*} \leftarrow \hat{\boldsymbol{\delta}}^{t}$.

- $\quad$ If $t=T$, then Print $\left(\hat{\boldsymbol{\delta}}^{*}, \gamma^{*}\right)$ and halt.

Master problem:

- Compute estimated values

$$
\mathbf{V}^{t} \equiv\left(\mathbf{V}^{\text {Gen }, t}, \mathbf{V}^{\text {Line }, t}, \mathbf{V}^{\text {Bus }, t}, \mathbf{V}^{\text {Sub }, t}\right)=
$$$$
\frac{1}{t} \sum_{t^{\prime}=1}^{t}\left(\mathbf{V}^{\text {Gen }, t^{\prime}}, \mathbf{V}^{\text {Line, }, t^{\prime}}, \mathbf{V}^{\text {Bus }, t^{\prime}}, \mathbf{V}^{\text {Sub }, t^{\prime}}\right) \text {. }
$$

- $\quad$ Solve $\operatorname{MP}\left(\mathbf{V}^{t}, \hat{\Delta}^{t}\right)$ for $\hat{\boldsymbol{\delta}}^{t+1}$.

- If $\operatorname{MP}\left(\mathbf{V}^{t}, \hat{\Delta}^{t}\right)$ is infeasible, then Print $\left(\hat{\boldsymbol{\delta}}^{*}, \gamma^{*}\right)$ and halt.

- Update $\hat{\Delta}^{t+1} \leftarrow \hat{\Delta}^{t} \cup\left\{\hat{\boldsymbol{\delta}}^{t+1}\right\}$.

- $\quad$ Set $t \leftarrow t+1$.

- Return to Subproblem.

\section{RESULTS}

\section{A. Implementation}

We have applied algorithm I-ALG to two test networks drawn from the 1996 IEEE Reliability Test System (RTS) [8]-[9]. Tests are carried out on a $1 \mathrm{GHz}$ personal computer with $1 \mathrm{~GB}$ of RAM. The model and algorithm are implemented in GAMS, which is an algebraic modeling language for numerical optimization problems. GAMS enables easy generation and manipulation of the subproblems and master problems, which are actually solved with CPLEX, a highly efficient linear- and integerprogramming code [10].

We restrict the number of iterations to $T=500$ for all problems to limit computation time. For fixed $M$, the most difficult problems require about 200 seconds, excluding model-generation overhead. About $90 \%$ of the time is spent solving the master problem.

Our model is driven by generation and shedding costs. In the examples, we assume a fixed per-unit penalty for unmet load so only a single customer class is represented. Shedding costs are much higher than generation costs, so we present results only in terms of load shed.

\section{B. Test-case description}

The RTS examples are not intended to represent particular systems but, rather, general reference grids that contain most of the technologies and configurations found in typical power grids [8]. The cases we study, One Area RTS-96 ("RTS1") and Two Area RTS-96 ("RTS2”), are described in detail in [8]. RTS2 duplicates RTS1 and adds three interconnections between the duplicated areas.

Interdiction data must be defined in addition to grid data. For these examples, we suppose that the terrorists' resources are quantified as a given number of people: One person is required to interdict any overhead single line or physically parallel overhead lines, although underground cables cannot be interdicted; transformers (which are represented by lines) require two people; three people are required to interdict any bus or substation; but generators are well-protected and cannot be attacked directly (although they can be disconnected from the grid by attacking the associated bus).

\section{Interdiction plans}

Figures 1 and 2 display the amount of load shed in each grid when total interdiction resource $M$ varies from zero to forty. The amount of load shed is a monotonically non-decreasing function of $M$, with a tendency to concavity as $M$ increases; this is to be expected. 


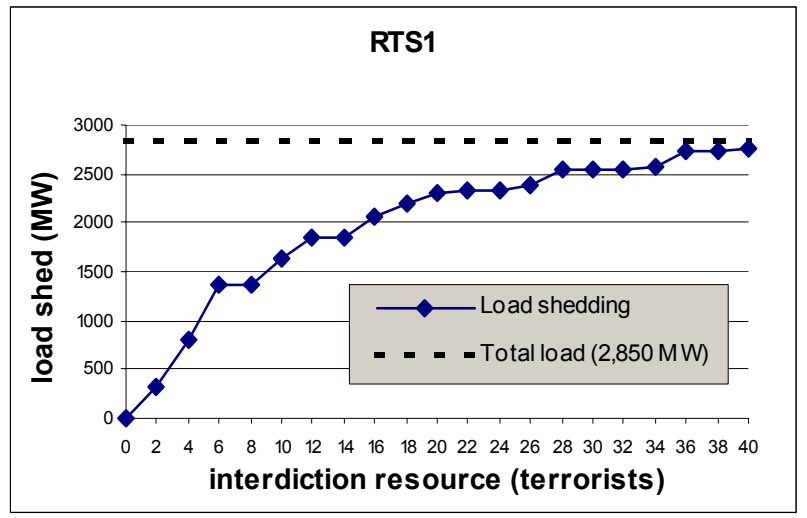

Figure 1: Load shedding for RTS1 as a function of interdiction resource $M$ (number of terrorists). Total load is $2,850 \mathrm{MW}$.

In RTS1, any case with $M \geq 28$ results in at least $90 \%$ of the total load being shed. For the most part, RTS2 is more difficult to interdict when compared to the RTS1 for twice the amount of interdiction resource. For example, 2,311 MW are shed in RTS1 when $M=20$, whereas only 4,000 MW are shed in RTS2 when $M=40$. The interconnecting lines may be playing an important role in decreasing the impact of the attacks. However, we observe the opposite effect when $M$ is small. For instance, there is little disruption that two terrorists can cause in RTS1 but four terrorists cause proportionately more disruption in RTS2 because they can, apparently, focus on the "weak links" of a single area. (We caution that these observations apply to this particular system and do not necessarily generalize.)

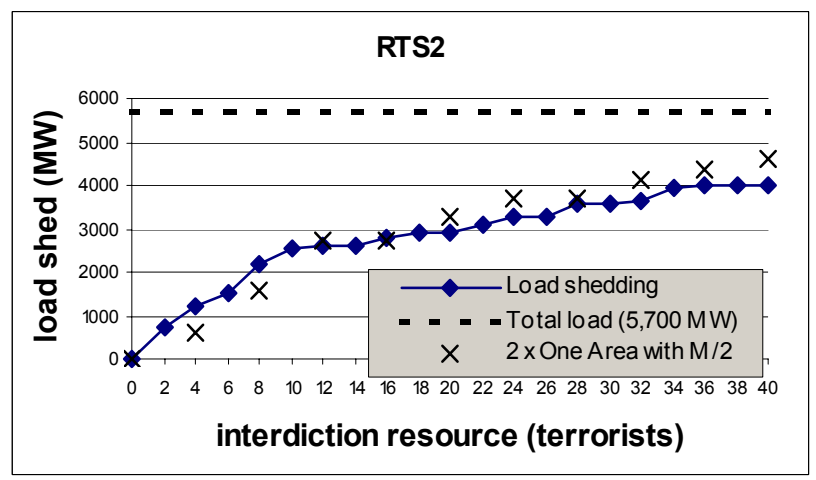

Figure 2: Load shedding for RTS2 as a function of interdiction resource $M$. Total load is 5,700 MW. Results are compared to twice the load shed in RTS1 with interdiction resource at $1 / 2 M$.

Determining likelihoods for each of these scenarios and incorporating them into a more elaborate stochastic program (e.g., [11], [12]) is possible, but is beyond the scope of this paper. It is instructive to compare two specific scenarios, however, and we do this next for RTS1.

Two near-best plans for RTS1, for $M=6$, are depicted in Figure 3. "Plan 1" attacks the substation and three selected lines, shedding 1,258 MW (44.1\% of the total load), and "Plan 2" attacks six selected transmission lines, shedding 1,373 MW (48.2\%). Plan 2 sheds more instantaneous load, but we must ultimately consider the total amount of unsupplied energy while the effects of the attack last. The $115 \mathrm{MW}$ of additional short-term load shedding in Plan 2 may be negligible compared to the long-term disruption caused by destroying the four transformers in Plan 1 , since it is unlikely those transformers could be replaced or repaired quickly. We investigate this issue more formally in the next section.

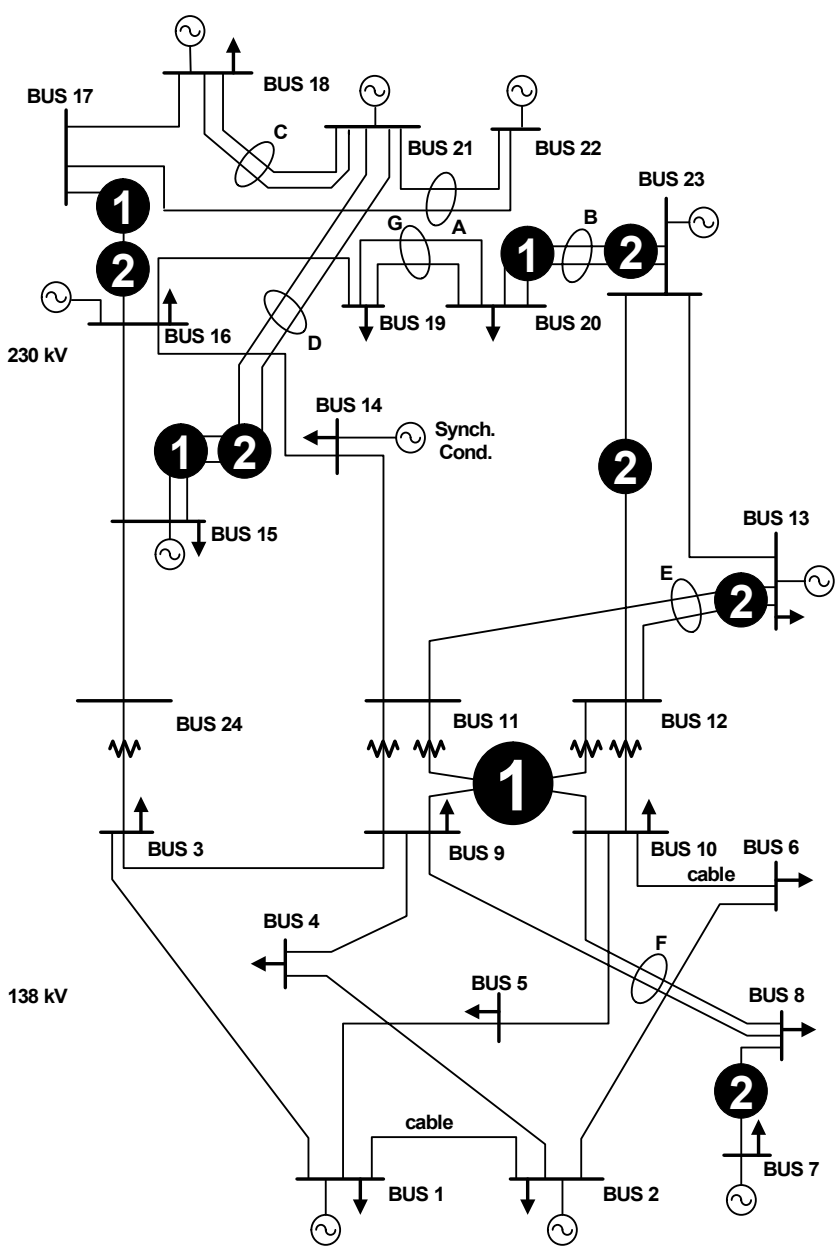

Figure 3: Two interdiction plans (depicted as $\mathbf{0}$ and (2) for RTS1 using $M=6$. Total load is $2,850 \mathrm{MW}$. Plan 1 sheds 1,258 MW and Plan 2 sheds 1,373 MW. The large "(-" indicates that the four transformers and buses in the substation are interdicted. 


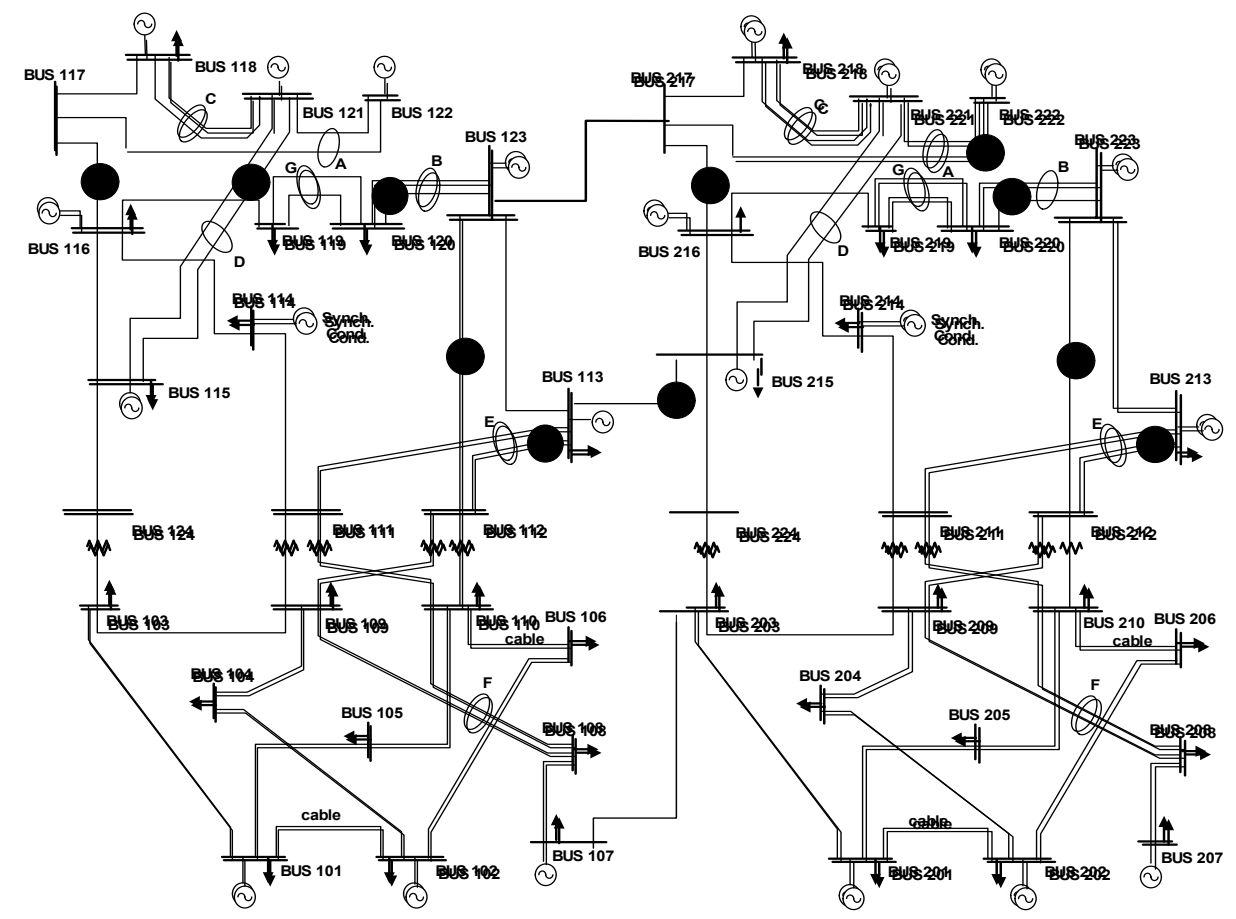

Figure 4: An interdiction plan ( $\bullet$ for RTS2 using $M=12$. Total load is 5,700 MW. This plan sheds 2,516 MW.

We now consider RTS2. Figure 4 depicts the most disruptive attack plan found to affect instantaneous load shedding for $M=12$. Here, $2,516 \mathrm{MW}$ (44.1\% of total load) is shed. Thus, doubling interdiction resource from $M=6$ in RTS1 to $M=12$ here yields not quite twice the disruption $(2,516<2 \times 1,373)$; compare Figure 2 . The RTS2 plan partially duplicates Plan 1 for RTS1, which seems sensible since the RTS2 duplicates RTS1's structure. Note, however, that one line connecting the duplicated areas is also interdicted.

\section{Results including restoration over time}

A time-phased version of I-DC-OPF is created by using interdiction constructs to couple instances of DC-OPF, one for each system state that represents a stage or "time period" of system repair. In outline, the model is:

$$
\begin{aligned}
&\left(\mathrm{Mm}^{\prime}\right) \quad \max _{\boldsymbol{\delta} \in \Delta} \min _{\mathbf{p}_{t}, \tau=1, \ldots \mathrm{T}} \sum_{\tau=1}^{\mathrm{T}} \alpha_{\tau} \mathbf{c}^{T} \mathbf{p}_{\tau} \\
& \text { s.t. } \mathbf{g}_{\tau}\left(\mathbf{p}_{\tau}, \boldsymbol{\delta}\right) \leq \mathbf{b} \quad \tau=1, \ldots, \mathrm{T} \\
& \mathbf{p}_{\tau} \geq \mathbf{0} \quad \tau=1, \ldots, \mathrm{T} .
\end{aligned}
$$

Model ( $\left.\mathrm{Mm}^{\prime}\right)$ extends $(\mathrm{Mm})$ to incorporate the hourly cost of power flow, $\mathbf{c}^{T} \mathbf{p}_{\tau}$, in each time period $\tau$, multiplied by the period's duration in hours, $\alpha_{\tau}$. The model could be extended to incorporate "sub-time periods" through load duration curves, be we have not yet explored this possibility; all loads are held constant over time. The conversion of I-ALG to solve ( $\left.\mathrm{Mm}^{\prime}\right)$ is straightforward.
Data for outage durations (i.e., repair or replacement times) are based loosely on [8]. Outage duration for transformers is 768 hours. For overhead lines, instead of the 10 or 11 hours used in [8], we are more conservative and assume 72 hours. This is justified because (a) we expect more damage to result from the intentional destruction of a line - this would probably involve the destruction of one or more towers [13] - than the average time needed to repair damage from common natural causes such as lightning, and (b) if $n$ lines and other grid elements are attacked, total repair time may be longer if fewer than $n$ repair teams are available. We also assume that a large substation requires 768 hours for repair, but buses, for which [8] provides no data, require 360 hours. These data are summarized in Table 1.

Table 2 presents results for each time period for attack Plans 1 and 2 in $\operatorname{RTS} 1(M=6)$. Immediately after the attack, Plan 2 sheds more power (1,373 MW) than Plan 1 $(1,258 \mathrm{MW})$. But, with power restoration over time factored in, Plan 1 causes more disruption because repairs take longer and more total energy is shed.

Table 1: Repair and interdiction data for grid components.

\begin{tabular}{|l|c|c|c|}
\hline $\begin{array}{c}\text { Grid } \\
\text { Component }\end{array}$ & $\begin{array}{c}\text { Inter- } \\
\text { dictable }\end{array}$ & $\begin{array}{c}\text { Resources } \boldsymbol{M} \\
\text { (\# of terrorists) }\end{array}$ & $\begin{array}{c}\text { Outage } \\
\text { Duration (h) }\end{array}$ \\
\hline Lines (overhead) & YES & 1 & 72 \\
\hline Lines (undergnd) & NO & N/A & N/A \\
\hline Transformers & YES & 2 & 768 \\
\hline Buses & YES & 3 & 360 \\
\hline Generators & NO & N/A & N/A \\
\hline Substations & YES & 3 & 768 \\
\hline
\end{tabular}


As expected, the revised model incorporating outage durations identifies a more disruptive plan, Plan 3 (see Table 2 and Figure 5) in terms of total energy shed, compared to Plans 1 and 2. Plan 3 interdicts the large substation, a transformer in the other substation (but without disconnecting the load associated with the substation on the left) and one line.

Table 2: Energy shed until system repair is complete, for three attack plans for RTS1. Plan 3 is superior because it was generated through $\left(\mathrm{Mm}^{\prime}\right)$ which explicitly models power restoration over time.

\begin{tabular}{|c|c|r|r|}
\hline Plan & $\begin{array}{c}\text { Time } \\
\text { Period }\end{array}$ & $\begin{array}{c}\text { Power } \\
\text { Shed (MW) }\end{array}$ & $\begin{array}{c}\text { Energy } \\
\text { Shed (MWh) }\end{array}$ \\
\hline \multirow{3}{*}{1} & $0-72 \mathrm{~h}$ & 1,258 & 90,576 \\
\cline { 2 - 4 } & $72-768 \mathrm{~h}$ & 426 & 296,496 \\
\cline { 2 - 4 } & \multicolumn{3}{|c}{ Total: 387,072 } \\
\hline \multirow{2}{*}{2} & $0-72 \mathrm{~h}$ & 1,373 & 98,856 \\
\cline { 2 - 4 } & \multicolumn{3}{|c}{ Total: 98,856 } \\
\hline \multirow{3}{*}{3} & $0-72 \mathrm{~h}$ & 902 & 64,944 \\
\cline { 2 - 4 } & $72-768 \mathrm{~h}$ & 708 & 492,768 \\
\cline { 2 - 4 } & \multicolumn{3}{|c}{ Total: 557,712 } \\
\hline
\end{tabular}

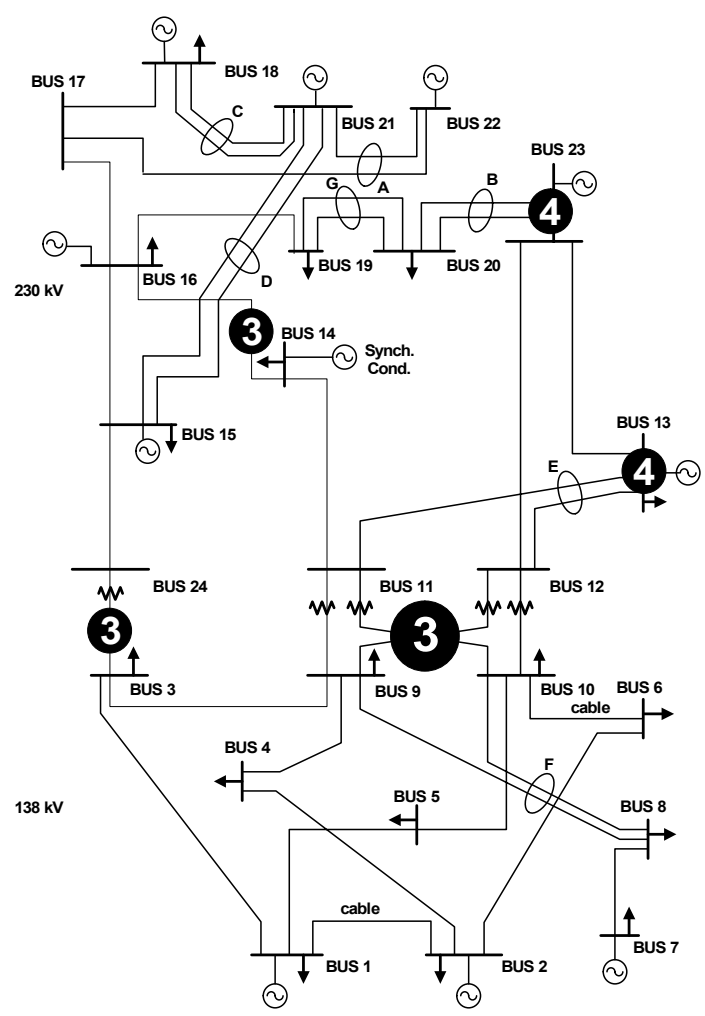

Figure 5: Interdiction Plans 3 (3) and 4 (4) for RTS1 with $M=6$ and explicit modeling of system restoration over time. Total energy shed is 557,712 MWh for Plan 3. Plan 4 (attack to two buses) is created assuming the two substations attacked in Plan 3 have been rendered invulnerable. Total energy shed drops to 272,160 MWh.

\section{E. Identifying candidate components for hardening}

Our ultimate goal is to identify grid components which, when "hardened," yield the best improvement in system security. A transmission line like the one attacked in Plan 3 would be difficult or impossible to harden, although a similar effect might be achieved by adding redundant capacity in a new, separate, parallel corridor. However, opening up new corridors for power lines is a slow, difficult and expensive process and a more practical alternative might be to harden the two substations that Plan 3 indicates are important. Indeed, when those two substations are treated as invulnerable, the best attack plan found, Plan 4 in Figure 5, sheds only 272,160 MWh of electricity compared to $557,712 \mathrm{MWh}$ for Plan 3. Hardening a substation could be accomplished in a number of ways, for example, by strengthening building walls or by enclosing outdoor facilities.

The components that are identified as candidates for hardening will naturally depend on the assumptions regarding $M$, i.e., the terrorists' assumed resources. If certain components appear to be critical over a wide range of values for $M$, then it would be reasonable to focus on those components for hardening. However, more research will be required to formalize an approach to this problem.

\section{CONCLUSIONS AND FUTURE WORK}

In this paper we have formulated the problem of optimal interdiction of an electric power network and have developed a heuristic algorithm to solve the problem. We have demonstrated the algorithm using two RTS systems and have indicated how the results can be used by planners to identify critical components whose hardening will substantially improve system security. Critical components are identified with modest computational effort.

Numerous issues remain for future work, and they include:

- Creating linear approximations of the model (Mm) having the form

$$
\begin{aligned}
&(\mathrm{LMm}) \quad \max _{\boldsymbol{\delta} \in \Delta} \min \boldsymbol{c}^{T} \mathbf{p} \\
& \text { s.t. } A \mathbf{p} \leq B \boldsymbol{\delta} \\
& \mathbf{p} \geq \mathbf{0},
\end{aligned}
$$

which are amenable to exact solution methods;

- Extending (Mm) and (LMm) to represent more detail about system restoration and unmet load over time;

- $\quad$ Extending $(\mathrm{Mm})$ and $(\mathrm{LMm})$ to

(PLMm) $\quad \min _{\psi \in \Psi} \mathbf{d}^{T} \psi+\max _{\boldsymbol{\delta} \in \Delta(\psi)} \min \mathbf{c}^{T} \mathbf{p}$

$$
\begin{aligned}
\text { s.t. } A(\psi) \mathbf{p} & \leq B(\psi) \boldsymbol{\delta} \\
\mathbf{p} & \geq \mathbf{0},
\end{aligned}
$$

where the new level of optimization over $\psi \in \Psi$ represents protective measures to be taken in advance, such as hardening particular grid components as discussed in section III.E. These measures will reduce 
the ability of terrorists to attack the grid through the constraints represented by $\delta \in \Delta(\psi)$, and thereby improve post-attack power flows;

- Incorporating uncertainty about terrorists' capabilities into our analysis;

- Evaluating different spare-equipment policies, including the use of "generic" transformer spares that could provide adequate, if imperfect, replacements for several types of transformers; and,

- Representing the immediate aftermath of an attack, including cascading outages and the dynamics of voltage collapse and angular instability.

\section{REFERENCES}

[1] Office of Technology Assessment (1990), "Physical Vulnerability of Electric Systems to Natural Disasters and Sabotage," OTA-E-453.

[2] National Energy Policy (2001), "Report of the National Energy Policy Development Group," Vicepresident's Task Force.

[3] Committee on Science and Technology for Countering Terrorism (2002). "Making the Nation Safer. The Role of Science and Technology in Countering Terrorism," National Research Council, National Academy Press, Washington, D.C.

[4] Qiu, B., Liu, Y., and Phadke, A. G. (2002). "Communication Infrastructure Design for Strategic Power Infrastructure Defense (SPID) System," Proceedings of the 2002 IEEE Power Engineering Society Winter Meeting, 27-31 January.

[5] Israeli, E. and Wood, K. (2002). "Shortest-Path Network Interdiction," Networks, Vol. 40, pp. 97111.

[6] A.J. Wood and B.F. Wollenberg (1996). Power Generation, Operation and Control. Second Edition. John Wiley and Sons, New York.

[7] Geoffrion, A.M. (1972). "Generalized Benders Decomposition," Journal of Optimization Theory and Applications, Vol. 10, pp. 237-260.

[8] IEEE Reliability Test Data (1999-I). "The IEEE Reliability Test System - 1996," IEEE Transactions on Power Systems, Vol. 14, pp. 1010-1020.

[9] IEEE Reliability Test Data (1999-II). Data from www.ee.washington.edu/research/pstca/.

[10] GAMS-CPLEX (2003). www.gams.com (accessed January, 2003).

[11]Cormican, K., Morton, D. and Wood, K. (1998). "Stochastic Network Interdiction," Operations Research, Vol. 46, pp. 184-197

[12] Birge, J. and Louveaux, F. (1997). Introduction to Stochastic Programming. Springer-Verlag, New York.

[13] Miami Herald (2002). "Rebel tactics cause concern," March 6, available from DTIC.

\section{ACKNOWLEDGMENTS}

This research is supported by the U.S. Department of Justice, Office of Justice Programs and Office of Domestic Preparedness (2002-GT-R-057), by the Office of Naval Research, and by the Air Force Office of Scientific Research.

Javier Salmeron received his M.S. and Ph.D. in Mathematics from Complutense University and Polytechnic University of Madrid, respectively. $\mathrm{He}$ is currently Research Assistant Professor in the Operations Research Department at the Naval Postgraduate School.

Kevin Wood received B.S. degrees in electrical engineering and mathematics from the University of Portland. He received his M.S. from Columbia University and his Ph.D. from University of California at Berkeley, both degrees in Operations Research. He is now Professor in the Operations Research Department at the Naval Postgraduate School.

Ross Baldick received his B.S. and B.E. from University of Sydney, Australia, and his M.S. and Ph.D. from the University of California, Berkeley. $\mathrm{He}$ is currently Associate Professor in the Department of Electrical and Computer Engineering at the University of Texas at Austin. 


\section{Response from authors:}

We would like to thank the reviewers for their comments, which have helped us to improve the paper considerably. In the following, we include the reviewers' comments in italics and our responses after each comment.

\section{Editor's Comments:}

Figures should be redrawn.

We have redrawn the figures.

\section{Reviewers' Comments:}

\section{Reviewer 1 Comments:}

General Comments and Changes:

The authors have written a timely article concerning the security of the power system against terrorist attacks. One aspect of the threat has been cast into an interdiction model that addresses the use of a limited set of resources to disable a portion of the power network. The analysis seeks to find the weaknesses in a network by maximizing load shedding for a fixed amount of attack resources. The power system analysis is static in this work.

Two aspects not mentioned in the paper (perhaps needed in the conclusions) are the possiblity of cascading failures in the system and the dynamic problems of voltage collapse and angular instability.

We have added another bulleted item to the future work describing this issue.

Although great effort was put in to writing the major equations in block form, readers of the paper would find the reading easier if the authors would place the equations within the body of the text.

We are unsure how to respond to this suggestion. Blocks of equations such as
(DC.0) to (DC.5) would be very unwieldy if incorporated into the text. Moreover, we refer to these equations explicitly by equation number in the subsequent text. Incorporating them into the body of the text would prevent us using equation numbers.

The authors are encouraged to add a few conference paper references from the past two to three PES meetings that have dealt with the security issues of the power system. Some of the authors who have worked in this area are Phadke, Heydt, and Ilic.

We searched the most recent three PES meetings (2002 Winter, 2002 Summer, and 2003) for these authors and for articles on security and found only one relevant paper, by Phadke. We have included this reference in the updated paper, thank the reviewer for drawing our attention to these authors, and ask for more specific reference to any papers that we should include in addition.

The conclusion is weak in that only future efforts are listed. Please include points that were discovered and draw some conclusions from the work that was performed.

We have added a paragraph to the conclusion outlining the contribution.

It is suggested that the authors review the IEEE PES formatting guidelines and make appropriate corrections to the tables and figures.

We have re-drawn the figures and moved the table captions to above the tables.

Specific Changes Required: Numbering of equations should be consistent with IEEE paper instructions. The use of compound alpha-numeric labels is not really necessary. 
We feel that the compound labels group relevant equations together. We request that we be permitted to leave the numbering as it is currently.

The first mentioning of the DC approximation appears on the first page in the second section at the end of the second paragraph. The definition of the DC approximation appears later in subsection $B$ on page 2. Although many of us are familiar with Wood's and Wollenberg's network simplification, the definition should appear at the first usage. I recommend that on the first page, the term "DC approximation" be changed to "The inner product is a simplified optimal power flow, which is linear in..."

We have made the suggested change and appreciate the careful reading!

The software packages GAMS and CPLEX are only mentioned in the implementation. As a minimum, the authors should give a short description of the type of software tools these are. It would be more useful to the readers of many years from now for the authors to give a general software approach.

We have added brief explanations of GAMS and CPLEX.

The network graphs, Figures 3, 4, and 5, appear grainy and the dotted number labels are not uniform in size. The figures should be enlarged for clarity. The figures appear to be taken from another work which compounds the graininess and poor quality. Consider redrawing the figures. If bus labels are to be included, they must be legible.

We have re-drawn the figures.
Reviewer 2 Comments:

General Comments and Changes:

This paper represents the beginning of an era in which power system engineers attempt to apply what we know about contingency analysis, OPF, security analysis etc to the problem of preparing for attacks against power systems by terrorists. Since terrorists can read, one certainly hopes they do not have the algorithmic sophistication required by this paper.

The data that terrorists would need to exploit our analytical methods are no longer easily available, right off the web. Thank goodness.

The paper also, I believe, leads into the obvious topic of "cost effective security analysis" in which one tries to pose the question of whether a power system should take protective actions in the face of possible contingencies when those actions will induce a large cost increase in the system operation.

We outline this extension in the "Conclusions and future work" section.

\section{Specific Changes Required:}

Reviewer 3 Comments:

General Comments and Changes:

This paper focuses on a very pertinent and timely topic. The authors have presented a mathematical approach to identifying those power system components that, if simultaneously outaged, would have dire consequences for the nation's bulk power system.

The authors provide a means of quantifying a measure of unserved load versus the amount of effort (number of terrorists) required to accomplish this goal. The authors provide guidance in both the amount of instantaneous outages, but also a measure of the duration of the outages.

Specific Changes Required: 
The primary drawbacks to this paper are:

1) the figures are unacceptable. The systems should be redrawn and not scanned.

We have redrawn the figures.

2) the authors do not address how the system resources should be "hardened." How does one harden a transmission line to make it invulnerable to attack? This is a major undertaking and should be addressed in the paper.

We agree that it is essentially impossible to harden a transmission line to make it invulnerable to attack. The real alternative with transmission lines is to add new ones along new corridors so that more effort is required of terrorists to reduce overall system capacity. We have added some discussion to this effect in section III.E. We have also added a sentence suggesting how one might harden a substation.

Reviewer 4 Comments:

General Comments and Changes:

This paper provides an interesting algorithm to measure the cost after initial restoration following terrorist attacks. A two level mathematical model is suggested to find the optimal interdiction plan given the total interdiction resource available to terrorists. A decomposition-based heuristic is implemented to find the "optimal" plan.

This is an important topic since power system security is receiving considerable attention these days. As the authors claimed, this paper is to introduce the analytical techniques to help mitigate the disruptions to electric power grids. However, the authors did not mention how to mitigate the disruptions according to the algorithm analytical results. For example, for different interdiction resource $M$, different optimal interdiction plans (different critical components) may be identified. The authors should further address how to determine which are most critical components and what kinds of measures could be taken to protect these critical components.

We discuss the identification of critical components in section III.E "Identifying candidate components for hardening" and outline some measures to harden substations. A general methodology to identify the optimal candidates for hardening is the subject of ongoing research.

Specific Changes Required: Required Changes:

\section{Figure 4 is not legible.}

We have redrawn the figures.

2. The title of a table should be located above the table contents. (Refer to the IEEE transactions requirements.)

We have moved the table captions to be above the table.

3. In section IV, only future work is discussed. So the title "IV. Conclusions and Future Work" is not appropriate.

We have added a paragraph of conclusions to this section.

4. $\quad$ Page 7, Column A, "Plan 2 sheds more power $(1,375 \mathrm{MW})$ than Plan

1" should be "Plan 2 sheds more power (1,373

$M W)$ than Plan 1

" (Refer to Table 2 of this paper.)

We have re-written the sentence to refer to Table 2 and have replaced 1,375 with 1,373 . 
